\title{
Intracoronary dipyridamole reduces the incidence of abrupt vessel closure following PTCA: a prospective randomised trial
}

M P Heintzen, U E Heidland, W J Klimek, M Leschke, M Kelm, B Schwartzkopff, E G Vester, C J Michel, B E Strauer

\begin{abstract}
Objectives-To investigate the effect of intracoronary dipyridamole on the incidence of abrupt vessel closure, myocardial infarction, necessity for bypass grafting, and death following percutaneous transluminal coronary angioplasty (PTCA).

Patients-Patients were randomly allocated to receive either conventional pretreatment (heparin $15000 \mathrm{IU}$ and aspirin $500 \mathrm{mg}$ intravenously) or additional intracoronary dipyridamole $(0.5 \mathrm{mg} /$ $\mathrm{kg}$ bodyweight). Dipyridamole was administered in 550 PTCA procedures (455 interventions in men, mean (SD) age 59.2 (8.4) years; 74 acute coronary syndromes), while conventional pretreatment was administered in 544 interventions (444 interventions in men 58.3 (7.9) years old; 81 acute coronary syndromes). In 53 interventions bail out stenting was performed for threatened abrupt vessel closure.

Results-Intracoronary dipyridamole significantly reduced the incidence of abrupt vessel closure (odds ratio $0.42 .95 \%$ confidence interval (CI) 0.22 to 0.79 ). While abrupt vessel closure occurred in $6.1 \%$ of interventions following conventional pretreatment, dipyridamole reduced the incidence to $2.5 \%$. Restricting the analysis to balloon angioplasty, this reduction was observed in patients with stable angina (odds ratio $0.49,95 \%$ CI 0.23 to 0.96 ) as well as in those with acute coronary syndromes (odds ratio $0.29,95 \%$ CI 0.09 to 0.87 ). Reduction of secondary end points in the dipyridamole treated patients failed to reach significance in the PTCA group.

Conclusions-Intracoronary dipyridamole before PTCA reduces the incidence of abrupt vessel closure following PTCA for stable angina and acute coronary syndromes.

(Heart 2000;83:551-556)
\end{abstract}

Keywords: dipyridamole; percutaneous transluminal coronary angioplasty; abrupt vessel closure

Abrupt vessel closure continues to represent the most dangerous hazard of percutaneous transluminal coronary angioplasty (PTCA). Despite anticoagulation and inhibition of platelet aggregation, abrupt vessel closure complicates coronary angioplasty in $2-8 \%$ of cases. $^{1-3}$ Intracoronary stenting and pharmaceutical blockade of the glycoprotein IIb/IIIa receptor reduce the incidence of abrupt vessel closure,${ }^{45}$ but cannot completely resolve the issue. Numerous predictors of abrupt vessel closure have been established including lesion characteristics, and clinical and angiographic predictors. ${ }^{6}$ Owing to local activation of the coagulation system and intracoronary thrombus formation, the use of PTCA in treating acute coronary syndromes such as unstable angina or acute myocardial infarction is associated with an excessively high risk of abrupt vessel closure. ${ }^{7-10}$

Angioplasty induced injury of the coronary artery (type III injury) is followed by the adhesion and aggregation of platelets leading to intracoronary thrombus formation. ${ }^{11}$ Apart from the degree of vessel wall injury, activation of the local coagulation system and coronary perfusion determine the extent of intracoronary thrombus formation..$^{12}$

Dipyridamole induces dilatation of coronary arteries at the microvascular site and prevents platelet aggregation. Its mechanism of action as an antithrombotic medication can be attrib- uted to different mechanisms. These include phosphodiesterase inhibition with consecutive intraplatelet cAMP accumulation, and inhibition of adenosine degradation resulting in the stimulation of platelet adenylate cyclase. There are conflicting data concerning the efficacy of dipyridamole as an antithrombotic agent in humans. In a prospective randomised investigation oral dipyridamole did not prevent acute complications following PTCA. ${ }^{14}$ However, Danchin and colleagues described a significant reduction of abrupt vessel closure with intravenous dipyridamole in a retrospective analysis. $^{15}$

Since oral or intravenous application of dipyridamole is associated with a high level of plasma protein binding (up to 99\%) which might counteract a sufficient local concentration at the coronary site, intracoronary application of dipyridamole was performed in this investigation. ${ }^{16}$ The aim of our study was to assess the effect of intracoronary dipyridamole on the incidence of abrupt vessel closure and additional secondary end points defined as myocardial infarction, necessity for bypass grafting, and death following PTCA.

\section{Methods}

PATIENTS

Over a 14 month period (April 1995 to May 1996) 1623 coronary interventions were performed. Patients presenting with chronic 
obstructive pulmonary disease and hypotension, defined as systolic blood pressure below $100 \mathrm{~mm} \mathrm{Hg}(\mathrm{n}=102)$, and patients receiving recanalisation of a completely occluded coronary artery in the setting of stable angina $(n=181)$, were completely excluded from our study. Patients treated with intracoronary stenting $(\mathrm{n}=179$, including 53 interventions in which stenting was performed following randomisation) or alternative techniques like coronary atherectomy or laser angioplasty $(\mathrm{n}=$ 67) were not included in the analysis. Thus, restricting the analysis to pure balloon angioplasty, 1094 interventions $(67.4 \%$ of all interventions performed during the study period) in 1082 patients were analysed. In a second step, analysis was performed on an intention to treat basis of 1147 interventions including the 53 interventions in which bailout stenting had been performed following randomisation. In 155 interventions PTCA was performed in the setting of acute coronary syndromes, including 51 dilatations for unstable angina (class III, class B and C, according to Braunwald $^{17}$ ), and 17 and 104 dilatations for acute myocardial infarction (defined as onset of symptoms within a period of less than 24 hours). The study protocol was reviewed and accepted by the local ethics committee. Written informed consent was obtained from all patients. Only two patients refused to participate in the study and were treated with techniques other than angioplasty (stent implantation, atherectomy).

RANDOMISATION AND CORONARY ANGIOPLASTY All of the patients included in our investigation were treated with intracoronary isosorbide dinitrate $(0.3-0.6 \mathrm{mg})$ directly after placement of the guiding catheter, and were then randomised to receive either conventional pretreatment consisting of heparin $15000 \mathrm{IU}$ and aspirin $500 \mathrm{mg}$ intravenously or an additional intracoronary application of dipyridamole $(0.5 \mathrm{mg} / \mathrm{kg}$ bodyweight). Treatment with either conventional medication or additional dipyridamole was not blinded. Intracoronary dipyridamole was administered in two boluses, by manual injection over a time period of one minute per bolus. The first half of the calculated dosage of dipyridamole was given before balloon inflation following placement of the guiding catheter; the second portion was administered after completion of balloon angioplasty. The randomisation was performed by the technical assistants of the catheter laboratory immediately before angioplasty by opening sealed envelopes indicating the pretreatment to be applied. Coronary angioplasty was performed according to the standard criteria of our department. ${ }^{18}$ Patients receiving recanalisation of a completely occluded artery were admitted to our study only, when PTCA was performed in the setting of acute coronary syndromes. Recanalisation of completely occluded coronary arteries in the setting of acute coronary syndromes was performed in 46 interventions (21 interventions after dipyridamole application, 25 recanalisations following conventional pretreatment). In 53 interven- tions (25 patients receiving dipyridamole, 28 patients receiving conventional pretreatment) intracoronary stent implantation was required for insufficient balloon angioplasty results or threatened abrupt vessel closure after randomisation had already been performed. These interventions were only included in the intention to treat analysis and not in the analysis that was restricted to pure balloon angioplasty. The opened envelops containing the information on the randomisation decision were removed.

Quantitative coronary angiography (QCA) (Kontron Cardio 500) of the target lesion was performed in all patients enrolled into the study, immediately before and after PTCA at primary intervention and at follow up angiography. The investigator performing QCA was unaware of the patient's treatment. The degree of stenosis (\%) was assessed in the angiographic view in which the stenosis appeared most severe. If PTCA was performed in different parts of the vessel only the stenosis with the highest degree was analysed. QCA was performed with the aid of electronic caliper measurements performed on selected magnified cineframes referring to the known diameter of the guiding catheter. Minimal lumen diameter $(\mathrm{mm})$, vessel diameter ( $\mathrm{mm})$, and stenosis length $(\mathrm{mm})$ were analysed. In patients receiving recanalisation of a completely occluded coronary artery for acute coronary syndromes, only minimal lumen diameter and degree of stenosis after PTCA and vessel diameter were analysed.

In order to exclude a potential impact of vasoactive substances on the outcome of this study, oral antianginal medication was discontinued at least 12 hours before coronary angioplasty as a part of the study protocol. Only patients presenting with acute coronary syndromes received intravenous heparin and isosorbide dinitrate infusion until the beginning of the PTCA procedure.

\section{STUDY END POINTS}

The primary study end point was defined as occurrence of symptomatic abrupt vessel closure following primary successful angioplasty occurring in the first 48 hours after the intervention (TIMI flow grade $0-1$ ). In all patients diagnosis of abrupt vessel closure was confirmed angiographically and instrumental recanalisation was attempted. Repeat angiography was performed in cases of severe angina or occurrence of ECG signs of ischemia, or both. Secondary study end points consisted of additional in-hospital complications including myocardial infarction, necessity for bypass grafting, death following PTCA, and restenosis at follow up. Myocardial infarction was defined as ECG signs of transmural ischaemia resulting in an ST segment elevation exceeding $0.2 \mathrm{mV}$ combined with an increase in serum creatine kinase $(\mathrm{CK})>200 \mathrm{U} / 1$ and a CK-MB fraction $>8 \%$ of total CK activity. In 763 interventions (72.9\% of all PTCA performances) angiographic follow up at a mean of 201 (62) days was available. Angiographic restenosis was defined as at least $50 \%$ stenosis of the target lesion on follow up angiogram. Target lesion 
Table 1 Clinical characteristics of patients

\begin{tabular}{lccc}
\hline & $\begin{array}{l}\text { Dipyridamole } \\
n=575\end{array}$ & $\begin{array}{l}\text { Conventional } \\
n=572\end{array}$ & $p$ Value \\
\hline Age (years) & $59.2(8.4)$ & $58.3(7.9)$ & 0.79 \\
Female sex (\%) & 17.3 & 18.4 & 0.63 \\
Clinical presentation & 476 & 463 & 0.49 \\
$\quad$ Stable angina & 20 & 31 & 0.11 \\
$\quad$ Unstable angina & 54 & 50 & 0.74 \\
$\quad$ Acute myocardial infarction & 42.6 & 43.2 & 0.85 \\
History of myocardial infarction (\%) & 169 & 170 & 0.92 \\
Extent of coronary artery disease & 209 & 186 & 0.19 \\
1 vessel disease & 172 & 188 & 0.25 \\
2 vessel disease & & & \\
3 vessel disease & 246 & 229 & 0.38 \\
& 134 & 133 & 0.98 \\
Vessel dilated & 140 & 154 & 0.29 \\
LAD & 30 & 28 & 0.86 \\
LCx & & & \\
RCA & & & \\
CABG & & & \\
\hline
\end{tabular}

Data presented as mean (SD) or number/percentage of patients. LAD, left anterior descending coronary artery; LCx, left circumflex coronary artery; RCA, right coronary artery; CABG, coronary artery bypass graft.

Table 2 Quantitative angiographic data

\begin{tabular}{lccc}
\hline & $\begin{array}{l}\text { Dipyridamole } \\
n=550\end{array}$ & $\begin{array}{l}\text { Conventional } \\
n=544\end{array}$ & $p$ Value \\
\hline MLD (mm) before PTCA & $1.11(0.41)$ & $1.03(0.43)$ & 0.37 \\
MLD (mm) after PTCA & $1.96(0.52)$ & $1.95(0.49)$ & 0.92 \\
Vessel diameter (mm) before PTCA & $3.01(0.66)$ & $3.01(0.60)$ & 0.99 \\
Vessel diameter (mm) after PTCA & $3.01(0.68)$ & $3.01(0.55)$ & 0.94 \\
Stenosis degree (\%) before PTCA & $63.2(13.0)$ & $65.8(14.5)$ & 0.27 \\
Stenosis degree (\%) after PTCA & $34.8(7.2)$ & $35.2(7.1)$ & 0.88 \\
Stenosis length (mm) & $7.2(3.4)$ & $7.7(3.4)$ & 0.08 \\
\hline
\end{tabular}

Data presented as mean (SD); MLD, minimal lumen diameter; PTCA, percutaneous transluminal coronary angioplasty.

revascularisation was defined as PTCA/stent or bypass surgery performed because of restenosis of the target lesion.

STATISTICAL ANALYSIS

All data are presented as mean (SD). Significance was accepted with values of $\mathrm{p}<0.05$. Discrete variables were compared using $\chi^{2}$ analysis. However, when the expected value of a cell was $<5$, Fisher's exact test was used. Continuous variables were analysed with the help of Student's $t$ test for independent samples. To assess the effect of intracoronary dipyridamole application on study end points, odds ratios and $95 \%$ confidence intervals (CI) were calculated. Statistical analysis was performed by means of PC-SAS software version 6.10 .

Table 3 Effect of intracoronary dipyridamole on abrupt vessel closure and secondary end points in an analysis restricted to balloon angioplasty

\begin{tabular}{|c|c|c|c|c|}
\hline & $\begin{array}{l}\text { Dipyridamole } \\
(n=550)\end{array}$ & $\begin{array}{l}\text { Conventional } \\
(n=544)\end{array}$ & $\begin{array}{l}\text { Odds ratio } \\
(95 \% \text { CI) }\end{array}$ & $p$ Value \\
\hline \multicolumn{5}{|l|}{ All patients } \\
\hline Abrupt vessel closure & $14(2.5 \%)$ & $33(6.1 \%)$ & $0.42(0.22$ to 0.79$)$ & 0.004 \\
\hline Acute myocardial infarction & $8(1.5 \%)$ & $17(3.1 \%)$ & $0.47(0.20$ to 1.10$)$ & 0.07 \\
\hline CABG & 0 & $3(0.6 \%)$ & & \\
\hline Exitus & $1(0.2 \%)$ & $2(0.4 \%)$ & & \\
\hline \multicolumn{5}{|l|}{ Stable angina } \\
\hline Abrupt vessel closure & $10(2.1 \%)$ & $20(4.3 \%)$ & $0.49(0.23$ to 0.96$)$ & 0.05 \\
\hline Acute myocardial infarction & $6(1.4 \%)$ & $11(2.5 \%)$ & $0.54(0.20$ to 1.50$)$ & 0.23 \\
\hline CABG & 0 & $2(0.4 \%)$ & & \\
\hline Exitus & $1(0.2 \%)$ & $1(0.2 \%)$ & & \\
\hline \multicolumn{5}{|l|}{ Acute coronary syndrome } \\
\hline Abrupt vessel closure & $4(5.5 \%)$ & $13(16.0 \%)$ & 0.29 (0.09 to 0.87$)$ & 0.04 \\
\hline Acute myocardial infarction & $2(2.7 \%)$ & $6(7.6 \%)$ & $0.34(0.07$ to 1.75$)$ & 0.28 \\
\hline CABG & 0 & $1(1.3 \%)$ & & \\
\hline Exitus & 0 & $1(1.3 \%)$ & & \\
\hline
\end{tabular}

Data presented as $\mathrm{p}$ values and odds ratios of the effect of intracoronary dipyridamole on primary and secondary end points.

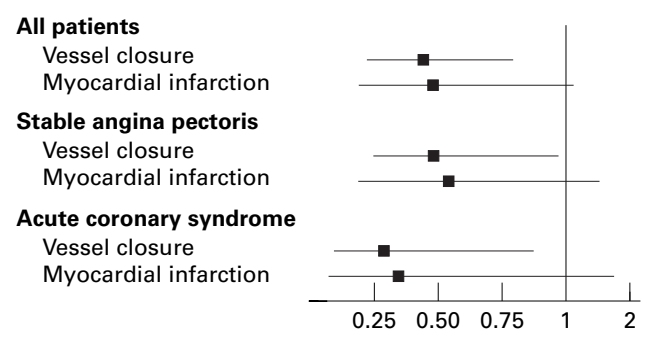

Figure 1 Effect of intracoronary dipyridamole on the incidence of abrupt vessel closure and acute myocardial infarction in an analysis restricted to balloon angioplasty. Odds ratio and 95\% CI of dipyridamole pretreatment and occurrence of abrupt vessel closure and myocardial infarction.

\section{Results}

DEMOGRAPHIC DATA

According to the randomisation intracoronary dipyridamole was administered in 550 interventions (455 interventions in men, 74 dilatations in the setting of acute coronary syndromes); in 544 dilatations conventional pretreatment was performed (444 interventions in men, 81 dilatations for acute coronary syndromes). There was no significant difference concerning age, sex, clinical presentation, history of myocardial infarction, and necessity of recanalisation in the setting of acute coronary syndromes (table 1). Furthermore, the extent of coronary artery disease, the vessel dilated, and data that were assessed by QCA (table 2) did not differ significantly.

INCIDENCE OF ABRUPT VESSEL CLOSURE

Abrupt vessel closure was observed in 47 interventions (table 3). Sixteen of these occlusions occurred while the patients were still in the catheter laboratory. Intracoronary dipyridamole pretreatment was associated with a highly significant prevention of abrupt vessel closure (fig 1), reducing the incidence in all interventions included from $6.1 \%$ (in those patients receiving conventional pretreatment) to $2.5 \%$ (odds ratio $0.42,95 \%$ CI 0.22 to 0.79 ). In dipyridamole pretreated patients four occlusions occurred in the catheter laboratory and 10 remote occlusions were noted. Following conventional pretreatment 12 occlusions occurred inside the catheter laboratory and 21 closures occurred after the patients departed. Analysing patients presenting with stable angina dipyridamole pretreatment resulted in a significant prevention, reducing the incidence of abrupt vessel closure by $50 \%$ (odds ratio $0.49,95 \%$ CI 0.23 to 0.96 ). In acute coronary syndromes prevention of abrupt vessel closure by intracoronary dipyridamole was even more pronounced (odds ratio $0.29,95 \%$ CI 0.09 to 0.87 ), reducing the incidence from $16.0 \%$ after conventional pretreatment to $5.5 \%$. Intention to treat analysis demonstrated a significant reduction of abrupt vessel closure in all patients and in the setting of stable angina. The reduction in the incidence of abrupt vessel closure failed to reach significance in acute coronary syndromes.

SECONDARY END POINTS

Following intracoronary administration of dipyridamole there was a notable reduction in 
Table 4 Effect of intracoronary dipyridamole on abrupt vessel closure and secondary end points in the intention to treat analysis

\begin{tabular}{lcclc}
\hline & $\begin{array}{l}\text { Dipyridamole } \\
n=550\end{array}$ & $\begin{array}{l}\text { Conventional } \\
n=544\end{array}$ & $\begin{array}{l}\text { Odds ratio } \\
(95 \% \text { CI })\end{array}$ & p Value \\
\hline All patients & $15(2.6 \%)$ & $34(5.9 \%)$ & $0.44(0.24$ to 0.80$)$ & 0.005 \\
$\quad$ Abrupt vessel closure & $8(1.4 \%)$ & $18(3.2 \%)$ & $0.44(0.19$ to 0.99$)$ & 0.05 \\
Acute myocardial infarction & 0 & $3(0.6 \%)$ & & \\
CABG & $1(0.2 \%)$ & $2(0.4 \%)$ & & 0.05 \\
Exitus & $10(2.0 \%)$ & $21(4.4 \%)$ & $0.47(0.22$ to 0.98$)$ & 0.16 \\
Stable angina & $6(1.2 \%)$ & $12(2.5 \%)$ & $0.49(0.19$ to 1.30$)$ & \\
Abrupt vessel closure & 0 & $2(0.4 \%)$ & & \\
Acute myocardial infarction & $1(0.2 \%)$ & $1(0.2 \%)$ & & 0.06 \\
CABG & $5(5.9 \%)$ & $13(14.6 \%)$ & $0.41(0.15$ to 1.09$)$ & \\
Exitus & $2(2.4 \%)$ & $6(6.7 \%)$ & $0.35(0.07$ to 1.70$)$ & 0.28 \\
Acute coronary syndrome & 0 & $1(1.3 \%)$ & & \\
Abrupt vessel closure & $1(1.3 \%)$ & & \\
Acute myocardial infarction & & & & \\
CABG & 0 & & & \\
Exitus & & & & \\
\hline
\end{tabular}

the incidence of myocardial infarction following PTCA which, however, failed to reach significance (odds ratio $0.47,95 \%$ CI 0.20 to 1.10). The incidence of bypass grafting and death were also less frequent after dipyridamole pretreatment, but again this failed to reach significance (table 3 ). Differential assessment of those patients presenting with stable ischemia and those presenting with acute coronary syndromes failed to detect significant differences concerning secondary end points in the analysis restricted to balloon angioplasty. However, intention to treat analysis (table 4) revealed a significant reduction in acute myocardial infarction in the entire group of patients following dipyridamole. Long term follow up, which was obtained in $72.9 \%$ of all interventions, revealed a trend towards a lower incidence of angiographic restenosis and target lesion revascularisation, but failed to reach significance (table 5).

SIDE EFFECTS OF INTRACORONARY DIPYRIDAMOLE APPLICATION

As in previous studies investigating the administration of intracoronary dipyridamole, ${ }^{19} 20$ drug associated side effects were rare and were recorded in just 11 patients $(2 \%)$. These included bradycardia $(n=3)$ caused by atrioventricular blockade following the administration of dipyridamole into the right coronary artery, and hypotension $(n=2)$ which required intravenous fluid administration. In six patients transient angina as a result of the coronary steal phenomenom was noted. All of these six patients presented with severe triple vessel coronary artery disease; in two of these patients PTCA of a bypass graft was performed. Intracoronary dipyridamole was not associated with an increase in bleeding complications at the puncture site.

Table 5 Effect of intracoronary dipyridamole on angiographic long term follow up

\begin{tabular}{llll}
\hline & $\begin{array}{l}\text { Dipyridamole } \\
n=375\end{array}$ & $\begin{array}{l}\text { Conventional } \\
n=388\end{array}$ & $p$ Value \\
\hline Restenosis (\%) & 36.8 & 43 & 0.08 \\
$\quad$ Stable angina & 34.6 & 41.9 & 0.06 \\
Acute coronary syndrome & 49.1 & 51.1 & 0.53 \\
TLR (\%) & 27.2 & 32.2 & 0.13 \\
Stable angina & 26.4 & 31.9 & 0.12 \\
Acute coronary syndrome & 31.6 & 34 & 0.41 \\
\hline
\end{tabular}

TLR, target lesion revascularisation.

\section{Discussion}

Our data indicate that intracoronary pretreatment with dipyridamole is associated with a highly significant reduction in the incidence of abrupt vessel closure. This prevention could be observed in patients with stable angina and in those with acute coronary syndromes. The comparatively high incidence of abrupt vessel closure, especially in the conventionally treated group, is explained by the high percentage of interventions for acute coronary syndromes accounting for $36.2 \%$ of all occlusions. However, in the analysis restricted to balloon angioplasty, patients presenting with acute coronary syndromes seemed to gain the greatest benefit from the addition of intracoronary dipyridamole. Secondary end points defined as myocardial infarction, necessity for bypass grafting, and death following PTCA were reduced by intracoronary dipyridamole but this reduction failed to reach significance. Only in the intention to treat analysis did the incidence of myocardial infarction demonstrate a significant reduction.

\section{IMPLICATIONS FOR INTRACORONARY DRUG} APPLICATION

There are conflicting data concerning the efficacy of dipyridamole in the prevention of abrupt vessel closure following PTCA. Barnathan and colleagues documented a prevention of abrupt vessel closure and a reduction of clinical asymptomatic intracoronary thrombus formation following intensive oral antithrombotic medication comprising aspirin and dipyridamole. ${ }^{21}$ In a prospective randomised trial Lembo and colleagues compared pretreatment with oral aspirin versus oral aspirin plus dipyridamole before PTCA. ${ }^{14}$ In this investigation additional oral application of dipyridamole failed to demonstrate a positive effect on complications following PTCA defined as myocardial infarction, necessity for bypass grafting, and death. In a retrospective analysis by Danchin and colleagues, intravenous infusion of dipyridamole $(30 \mathrm{mg})$ before PTCA was associated with a significant reduction of angiographically assessed abrupt vessel closure and myocardial infarction. ${ }^{15}$

Oral or intravenous application of dipyridamole is associated with a high degree of plasma protein binding (up to $99 \%$ ). ${ }^{16}$ This high level of plasma protein binding might counteract a sufficient local concentration of dipyridamole at the coronary site, which might explain the conflicting data concerning the efficacy of dipyridamole as an antithrombotic drug. Large dosages of dipyridamole are required to inhibit platelet aggregation in vitro. ${ }^{22}$ Thus the intracoronary application mode most likely represents the only possibility of achieving a sufficient dipyridamole induced platelet aggregation at the coronary site. Furthermore intracoronary infusion of dipyridamole is associated with a very low incidence of side effects. ${ }^{19} 20$

POSTULATED MECHANISM OF ACTION

The mechanism of action of dipyridamole in the setting of platelet aggregation in vivo is a subject of controversy. There are several 
different mechanisms contributing to the antithrombotic efficacy of dipyridamole. ${ }^{23}$ Dipyridamole inhibits the activity of phosphodiesterase leading to the degradation of cAMP. The increased intraplatelet cAMP concentration prevents platelet aggregation. ${ }^{24}$ Furthermore, it has been argued that dipyridamole exerts an antithrombotic effect by modulating the action of platelet inhibiting eicosanoids such as prostacyclin and prostaglandin D2. ${ }^{25}$ These substances lead to the inhibition of platelet aggregation and function by the direct stimulation of platelet adenylate cyclase. Inhibition of adenosine degradation represents an additional crucial mechanism, contributing to the antithrombotic effect of dipyridamole. ${ }^{26}{ }^{27}$ Under physiological circumstances adenosine is rapidly incorporated into erythrocytes and endothelial cells. ${ }^{28}{ }^{29}$ Dipyridamole inhibits adenosine reuptake leading to an increased local concentration of adenosine. Adenosine inhibits thrombocyte function by a direct stimulation of adenylate cyclase. ${ }^{30}$ The potential key role of the adenosine pathway in the antithrombotic efficacy of dipyridamole is stressed by Dawicki and colleagues. ${ }^{31}$ In this investigation small dosages of adenosine and dipyridamole, at concentrations which both compounds have no inhibitory effect on platelet function, were sufficient to suppress platelet aggregation in human blood. Adenosine exerts a pronounced vasodilating capacity at the microvascular site leading to an increase in coronary perfusion. This increase in blood flow might facilitate the dispersal of intracoronary thrombi and thus may represent an additional explanation of the antithrombotic effect of dipyridamole. Finally, dipyridamole may also inhibit platelet aggregation through a pharmacokinetic interaction with aspirin leading to an incremental additional antithrombotic effect. ${ }^{32}$ Pharmacokinetic interactions between aspirin and dipyridamole have been observed in the animal model. However, clinical studies have failed to document a mutual synergistic effect. $^{23}$

From our data it is not possible to elucidate completely the precise mechanism of action by which intracoronary dipyridamole prevents abrupt vessel closure. However, a synergistic effect involving adenosine induced coronary vasodilatation at the microvascular site combined with inhibition of thrombocyte aggregation represents the most convincing explanation of our findings.

STUDY LIMITATIONS

Abrupt vessel closure following PTCA can be caused by different mechanisms. These include intracoronary thrombus formation, development of an occlusive dissection, and vasoconstriction following PTCA. In some cases it remains impossible to clarify the exact cause of abrupt vessel closure using coronary angiography. As documented by intravascular ultrasound imaging, coronary angiography often represents a poor tool in the detection and classification of dissections following PTCA. Because of these methodological limitations our study does not investigate and compare the causal mechanisms leading to the development of abrupt vessel closure.

Another methodological limitation refers to the possibility of clinically "silent" vessel closure following PTCA. These cases would not have been included in our investigation. In an attempt to minimise the influence of clinically silent abrupt vessel closure, recanalisations of completely occluded coronary arteries in the setting of stable angina were not included in the study.

\section{CONCLUSIONS}

Intracoronary application of dipyridamole is associated with a highly significant reduction in the incidence of abrupt vessel closure following PTCA. This beneficial effect was observed in patients presenting with stable angina and acute coronary syndromes. However, in the analysis restricted to PTCA, patients receiving coronary angioplasty in the setting of myocardial infarction or unstable angina seem to gain the greatest benefit from intensifying the antithrombotic medication by intracoronary dipyridamole. In the intention to treat analysis myocardial infarction following PTCA was observed less frequently following intracoronary dipyridamole.

1 Detre KM, Holmes DR, Holubkov R, et al. Incidence and consequences of periprocedural occlusion: The 1985-1986 National Heart, Lung and Blood Institute percutaneous transluminal coronary angioplasty registry. Circulation 1990;82:739-50

2 Feyter de PJ, van den Brand M, Laarman GJ, et al. Acute coronary artery occlusion during and after percutaneous transluminal coronary angioplasty. Circulation 1991;83: 927-36.

3 Heintzen MP, Motz W, Leschke M, et al. Akuter Koronargefä $\beta$ verschlu $\beta$ nach elektiver perkutaner translu5000 konsekutiven Patienten. Dtsch Med Wschr 1994;119: 1023-8.

4 The Benestent Study Group. A comparison of balloonexpandable-stent implantation with balloon angioplasty in patients with coronary artery disease. N Engl f Med 1994; patients with

5 The EPIC Investigators. Randomised trial of coronary intervention with antibody against platelet IIb/IIIa integrin or reduction of clinical restenosis: results at six months. Lancet 1994;343:881-6.

6 Ellis SG, Vandormael MG, Cowley MJ, et al. Coronary morphologic and clinical determinants of procedural outcome with angioplasty for multivessel coronary disease. Implications for patient selection Circulation 1990;82: 1193-202.

7 Mabin TA, Holmes DR, Smith HC, et al. Intracoronary thrombus: role in coronary occlusion complicating percutaneous transluminal coronary angioplasty. $7 \mathrm{Am}$ Coll Cardiol 1985;5:198-202.

8 Chesler E, Gornick C, Pierpont G, et al. High incidence of acute coronary occlusions complicating percutaneous transluminal coronary angioplasty for angina pectoris. $\mathrm{Am}$ transluminal coronary angiol $1989 ; 15: 665-7$

9 Feyter de PJ, Serruys PW, vd Brand M, et al. Percutaneous Feyter de PJ, Serruys PW, vd Brand $\mathrm{M}$, et al. Percutaneous
transluminal coronary angioplasty for unstable angina. $\mathrm{Am}$ transluminal coronary angiop

10 Lincoff AM, Popma JJ, Ellis SG, et al. Abrupt vessel closure complicating coronary angioplasty: clinical, angiographic and therapeutic profile. $\mathcal{F}$ Am Coll Cardiol 1992;19:926-35.

11 Stein B, Fuster V, Israel DH, et al. Platelet inhibitor agents in cardiovascular disease: an update. $\mathcal{F} \mathrm{Am}$ Coll Cardiol 1989;14:813-36

12 Fernandez-Ortiz A, Badimon JJ, Falk E, et al. Characterization of relative thrombogenicity of atherosclerotic plaque components: implications for consequences of plaque rupture. F Am Coll Cardiol 1994;23:1562-9.

13 Merino A, Cohen M, Badimon JJ, et al. Synergistic action of severe wall injury and shear forces on thrombus formation in arterial stenosis: definition of a thrombotic shear rate threshold. $f$ Am Coll Cardiol 1994;24:1091-4.

14 Lembo NJ, Black AJR, Roubin GS, et al. Effect of pretreatment with aspirin plus dipyridamole on frequency and type of acute complications of percutaneous translumina coronary angioplasty. Am $\mathcal{f}$ Cardiol 1990;65:422-6. 
15 Danchin N, Juilliere Y, Kettani C, et al. Effect on early acute occlusion rate of adjunctive antithrombotic treatment with intravenously administered dipyridamole during percutaneous transluminal coronary angioplasty. Am Heart $\mathcal{f}$ 1994;127:494-8.

16 Kübler W. Die Bindung des Koronardilatators Dipyridamol an die Plasmaeiweißkörper des Menschen. Arch Kreislauf forsch 1971;64:115-26.

17 Braunwald E. Unstable angina: a classification. Circulation 1989;80:410-14.

18 Heintzen MP, Motz W, Leschke M, et al. PTCA im Stadium des akuten Myokardinfarktes: Hospitalverlauf von 785 konsekutiven Patienten. Z Kardiol 1994;83:404-13.

19 Strauer BE, Heidland UE, Vogt M, et al. Protektion und "Preconditioning" des menschlichen Herzens während perkutaner transluminaler Koronarangioplastie (PTCA) durch Dipyridamol intrakoronar. Med Klimik 1995;90:12530 .

20 Strauer BE, Heidland UE, Heintzen MP, et al. Pharmacologic myocardial protection during percutaneous translulogic myocardial protection during percutaneous translu-
minal coronary angioplasty by intracoronary application of minal coronary angioplasty by intracoronary application of
dipyridamole: Impact on hemodynamic function and left dipyridamole: Impact on hemodynamic function and left ven

21 Barnathan ES, Schwartz JS, Taylor L, et al. Aspirin and dipyridamole in the prevention of acute coronary thrombosis complicating coronary angioplasty. Circulation 1987;76: $125-34$.

22 Emmons PR, Harrison MJG, Honour AJ, et al. Effect of dipyridamole on human platelet behaviour. Lancet 1965;ii: 603-6.
23 Fitzgerald GA. Drug therapy: dipyridamole. $N$ Engl $\mathcal{F} \mathrm{Med}$ 1987;316:1247-57.

4 Mills DCB. The role of cyclic nucleotides in platelets. In: Kebabian JW, Nathanson JA, eds. Cyclic nucleotides: Part II: physiology and pharmacology. Berlin: Springer Verlag, 1982:723-61.

25 Moncada S, Korbut R. Dipyridamole and other phosphodiesterase inhibitors act as antithrombotic agents by potentiating endogenous prostacyclin. Lancet 1978;i:1286-9.

26 German DC, Kredich NM, Bjornsson TD. Oral dipyridamole increases plasma adenosine levels in human beings. Clin Pharmacol Ther 1989;45:80-4.

27 Kübler W, Spieckermann PG, Bretschneider HJ. Influence of dipyridamole (Persantin) on myocardial adenosine metabolism. F Mol Cell Cardiol 1970;1:23-37.

28 Olsson RA, Pearson JD. Cardiovascular purinoceptors. Physiol Rev 1990;70:761-845.

29 Schrader J, Berne RM, Rubio T. Uptake and metabolism of adenosine by human erythrocyte ghosts. Am f Physiol adenosine by huma

30 Haslam RJ, Lynham JA. Activation and inhibition of blood platelet adenylate cyclase by adenosine or by 2-chloradenosine. Life Sci 1972;11(pt 2):1143-54

31 Dawicki DD, Agarwal KC, Parks RE. Role of adenosine uptake and metabolism by blood cells in the antiplatelet actions of dipyridamole, dilazep and nitrobenzylthioinosine. Biochem Pharmacol 1985;34:3965-72.

32 Buchanan MR, Rosenfeld J, Gent M, et al. Increased dipyridamole plasma concentrations associated with salicylate administration: relationship to effects on platelet aggregation in vivo. Thromb Res 1979;15:813-20.

\section{IMAGES IN CARDIOLOGY}

\section{Effusive-constrictive pericarditis}

A 51 year old man presented with progressive worsening of chronic ascites. He had a history of chronic pericarditis of unknown cause requiring pericardiocentesis three timed in the last decade; the last performed six years previously.

At admission, physical examination revealed signs of systemic venous congestion, hepatomegaly, and a voluminous ascites with umbilical hernia. Spiral computed tomography of the heart after intravenous injection of contrast medium showed a mild amount of pericardial fluid, diffuse thickening, and calcification of visceral pericardium (epicardium), and only focal calcifications of parietal pericardium (the white arrows outline the thickened and calcified visceral pericardium; PE, pericardial effusion; $\mathrm{F}$, epicardial fat; RA, right atrium; RV, right ventricle; LA, left atrium; LV, left ventricle).

Right cardiac catheterisation was consistent with constrictive pericarditis. The definite diagnosis was chronic effusive-constrictive pericarditis. This condition is characterised by the coexistence of epicardial constriction and pericardial effusion. Pericardial fluid may cause cardiac tamponade in which case pericardiocentesis results in only partial and temporary relief.

Effusive-constrictive pericarditis appears to begin as an active effusive pericarditis, with

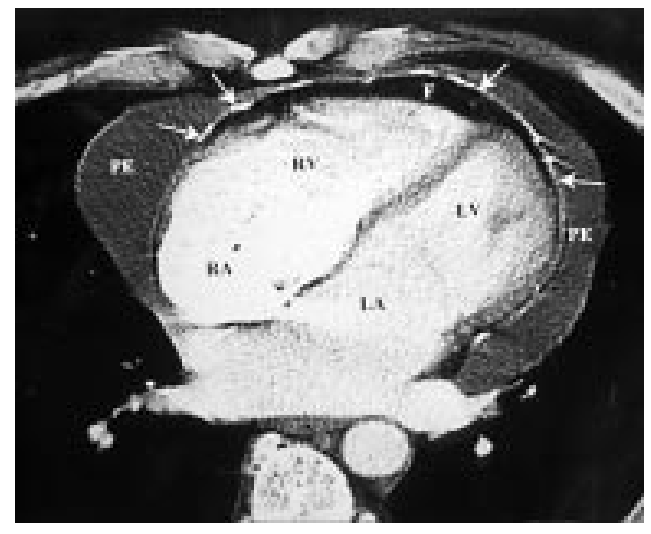

epicardial fibrosis and calcification developing later as a complication of the healing process. Reported causes do not seem to differ from those of non-effusive forms; the only exception being postsurgical constrictive pericarditis, which usually develops without effusion. Our patient had successful surgical removal of an extensively calcified epicardial layer.

MAURO SANTARONE GIOVANNI CORRADO GIANMARCO BELLONI 